Wissenschaftliche Taschenbücher

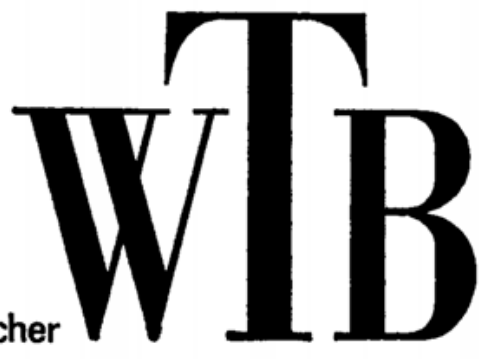

Chemie

\title{
Adolf Zschunke
}

Kernmagnetische Resonanzspektroskopie in der organischen Chemie

Akademie-Verlag · Berlin

Pergamon Press · Oxford

Vieweg + Sohn $\cdot$ Braunschweig 
ROLF BORSDORF / MAN FRED SCHOLZ

Spektroskopische Methoden in der organischen Chemie

WER NER HABERDITZL

Magnetochemie

GERHARD HEB ER

Mathematische Hilfsmittel der Physik, Teil I und II
A. A. SOKOLOW
Elementarteilchen
HEIN Z AHRENS
Varianzanalyse

HANS-J URGEN TREDER

Relativität und Kosmos

Raum und Zeit in der Physik, Astronomie und Kosmologie

ALBERT EINSTEIN

Grundzüge der Relativitätstheorie

ALBERT EINSTEIN

Über die spezielle und die allgemeine Relativitätstheorie

G UNTHER LUDWIG

Wellenmechanik. Einführung und Originaltexte

HARRY PAUL

Lasertheorie, Teil I und II

FRANZ RUDOLF KESSLER

Einführung in die physikalischen Grundlagen

der Kernenergiegewinnung

EBERHARD HOFMAN N

Eiweiße und Nucleinsäuren als biologische Makromoleküle

Dynamische Biochemie, Teil I

EBERHA R TEUSCHER

Pharmakognosie, Teil I und II

D. TER HAAR

Quantentheorie. Einführung und Originaltexte 
J. H. SAN DER S

Die Lichtgesehwindigkeit.

Einführung und Originaltexte

JEAN KUNTZMAN N

Unendliche Reihen

Mathematische Hilfsmittel der Physik und Chemie Mit 94 Utbungen und 29 Aufgaben

JEAN KUNTZMAN N

Systeme von Differentialgleichungen

Mathematische Hilfsmittel der Physik und Chemie Mit 88 Ubungen und 40 Aufgaben

JEAN KUNTZMAN N

Komplexe Veränderliche

Mathematische Hilfsmittel der Physik und Chemie Mit 90 Utbungen und 37 Aufgaben.

FER DINAND CAP

Einführung in die Plasmaphysik

I. Theoretische Grundlagen

- insgesamt 3 Bände -

J. A. ROSANOW

Wahrseheinliehkeitstheorie

HARRY PFEIFER

Theorie linearer Bauelemente

Elektronik für den Physiker I

HARRY PFEIFER

Die Elektronenröhre

Elektronik für den Physiker II

HARRY PFEIFER

Schaltungen mit Elektronenröhren

Elektronik für den Physiker III

HARRY PFEIFER

Leitungen und Antennen

Elektronik für den Physiker IV

HARRY PFEIFER

Mikrowellenelektronik

Elektronik für den Physiker V 
HARRY PFEIFER

Halbleiterelektronik

Elektronik für den Physiker VI

SIEG FRIE D HA U PTMAN N

Uber den Ablauf organisch-chemischer Reaktionen

GERHARD HUBNER / KLAUS JUNG / ECKART WINKLER

Die Rolle des Wassers in biologisehen Systemen

STEPHEN G. BRUSH

Kinetische Theorie, Teil I und II

EBERHARD HOFMAN N

Enzyme und energieliefernde Stoffwechselreaktionen

Dynamische Biochemie, Teil II

HERBERT GOERIN G

Elementare Methoden

zur Lösung von Differentialgleichungsproblemen

PETER KR UMBIEGEI.

Isotopieeffekte

D. M. B RIN K

Kernkräfte. Einführung und Originaltexte

DIETER ONKEN

Steroide

Zur Chemie und Anwendung .

HEINZ GEILER

Ökologie der Land- und Süßwassertiere

ARTH UR P. CRACKNELL

Angewandte Gruppentheorie. Einführung und Originaltexte

DIETER KLAUA

Elementare Axiome der Mengenlehre

EBERHAR D HOFMAN N

Intermediärstoffwechsel

Dynamische Biochemie, Teil III

G UNTER TEMBROCK

Grundlagen der Tierpsychologie

J. F. VINSON

Optische Kohärenz in der klassischen Theorie und in der Quantentheorie 
W. R. HIN D MAR SH

Atomspektren. Einführung und Originaltexte

G ÛNTER TEMBR O CK

Biokommunikation

Informationsübertragung im biologischen Bereich

Teil I und II

A D OLF ZSCHUN K E

Kernmagnetische Resonanzspektroskopie

in der organischen Chemie

Vorschau auf die nächsten Bände:

DIETER MERKEL

Riechstoffe

J OH N CUNN IN GHAM

Vektoren

FER DINAND CAP

Einführung in die Plasmaphysik

III. Magnetohydrodynamik

EBERHAR D H OFMAN N

Grundlagen der Molekularbiologie und Regulation

des Zellstoffwechsels

Dynamische Biochemie, Teil IV

GEORG DAUTCOURT

Relativistische Astrophysik 



\section{WTB}

BAND 38

Adolf Zschunke

Kernmagnetische

Resonanzspektroskopie in der organischen Chemie

Mit 81 Abbildungen und 37 Tabellen

AKADEMIE-VERLAG - BERLIN

PERGAMON PRESS - OXFORD

VIEWEG + SOHN - BRAUNSCHWEIG 


\section{Reihe CHEMIE}

Herausgegeben im Auftrage der Sektion Chemie

bei der Deutschen Akademie der Wissenschaften

von:

Prof. Dr. H. Klare, Teltow-Seehof

Prof. Dr.-Ing. Dr. h. c. E. Leibnitz, Berlin

Prof. Dr.-Ing. habil., Dr. rer. nat. h. c. K. Schwabe, Dresden

Prof. Dr. Dr. h. c. E. Thilo, Berlin

Verantwortlicher Herausgeber dieses Bandes:

Prof. Dr.-Ing. habil., Dr. rer. nat. h. c. K. Schwabe

Verfasser:

Dr. Adolf Zschunke

Martin-Luther-Universität, Halle - Wittenberg

ISBN $3528 \quad 060883$

\section{1}

Copyright 1972 by Akademie -Verlag GmbH, Berlin

Lizenznummer: $202 \cdot 100 / 572 / 71$

Herstellung: VEB Druckhaus „Maxim Gorki“, 74 Altenburg

Bestellnummer: Akademie-Verlag 7088 - EDV-Nummer: 7614907

Pergamon Press $08017597 \times$

Vieweg + Sohn 6088

Printed in German Democratic Republic 